Result(s)* 40 of 98 centres replied to the survey. $78 \%$ consented to become involved in an international registry. $63 \%$ of centres who replied receive between $50->100$ referrals of ovarian cancer per year and $90 \%$ perform HIPEC in ovarian cancer. $79 \%$ (31/40) stated they had been practising CRS \& HIPEC for $>10$ years. The number of CRS with HIPEC per year performed was $<20$ cases in $56 \%$ of respondents and $>50$ in $4 \%$.

$21 \%$ of centres held international accreditation for ovarian cancer CRS and 51\% held national accreditation. Of interest was that $82 \%$ reported that no certification was required for the administration of HIPEC in their country.

Conclusion* Given the multiple reports demonstrating variations in practice across the globe we believe this could be a very important opportunity for implementing change for women with ovarian cancer. For centres that consented to participation a further survey will be issued focusing on operating standards for CRS in ovarian cancer and protocols for HIPEC administration.

\section{BLOOD-BASED DETECTION OF CIRCULATING DICKKOPF- 1 AS A PROGNOSTIC BIOMARKER IN OVARIAN CANCER PATIENTS}

1,2 $\mathrm{P}$ Wimberger*, ${ }^{1,2} \mathrm{DM}$ Klotz, ${ }^{1,2} \mathrm{~T}$ Link, ${ }^{1,2} \mathrm{M}$ Goeckenjan, ${ }^{3} \mathrm{~N}$ Jaschke, ${ }^{3} \mathrm{LC}$ Hofbauer, ${ }^{3}$ A Göbel, ${ }^{3}$ TD Rachner, ${ }^{1,2} \mathrm{JD}$ Kuhlmann. ${ }^{1}$ Technische Universität Dresden, Department of Gynecology and Obstetrics, Dresden, Germany; ${ }^{2}$ National Center for Tumor Diseases, Partner site Dresden (NCT), Dresden, Germany; ${ }^{3}$ Technische Universität Dresden, Division of Endocrinology, Diabetes, and Bone Diseases, Department of Medicine III

\subsection{6/ijgc-2021-ESG0.511}

Introduction/Background* Dickkopf-1 (DKK-1) is a secreted protein, known for suppressing the differentiation and activity of bone-building osteoblasts by acting as an inhibitor of Wntsignalling. Soluble DKK-1 (sDKK-1) has been proposed as prognostic biomarker for a wide range of malignancies, however, clinical relevance of sDKK-1 as potential blood-based marker for ovarian cancer is unknown.

Methodology sDKK-1 levels were quantified in a cohort of 150 clinically documented ovarian cancer patients by a commercially available DKK-1 ELISA (Biomedica, Vienna, Austria). Result(s)* Median sDKK-1 level was significantly elevated at primary diagnosis of ovarian cancer compared to healthy controls (estimated difference (ED) of $7.75 \mathrm{ng} / \mathrm{mL}$ (95\%CI: 3.01 - $12.30 \mathrm{ng} / \mathrm{mL}, \mathrm{p}=0.001)$ ). Higher levels of sDKK-1 at diagnosis indicated an increased volume of intraoperative malignant ascites (ED $7.08 \mathrm{pmol} / \mathrm{L}, 95 \% \mathrm{CI}$ : 1.46 - 13.05, p $=0.02$ ) and predicted suboptimal debulking surgery (ED 6.88 pmol/L, 95\%CI: 1.73 - 11.87, p = 0.01). sDKK-1 did not correlate with CA125, and higher sDKK-1 levels predicted a higher risk of recurrence and poor survival (PFS: HR = $0.507,95 \% \mathrm{CI}: 0.317-0.809 ; \mathrm{p}=0.004$; OS: $\mathrm{HR}=0.561$, 95\%CI: $0.320-0.986 ; p=0.044)$. Prognostic relevance of sDKK-1 was partly sustained in wtBRCA patients (PFS: HR = $0.507,95 \%$ CI: $0.317-0.809$; $\mathrm{p}=0.004)$.

Conclusion* This is the first study demonstrating the prognostic relevance of sDKK-1 in ovarian cancer patients, including those with wtBRCA1/2 status. Our data encourage further evaluation of sDKK-1 in ovarian cancer patients, possibly in terms of a therapy monitoring marker or a response predictor for sDKK-1-directed targeted therapies.

\section{ROLE OF CA 125, HE4 AND IOTA SIMPLE RULES IN DIFFERENTIATING BENIGN AND MALIGNANT OVARIAN TUMOUR}

A Bahadur, R Mundhra*, N Bhattacharya. aiims rishikesh, obs gynae, rishikesh, India

\subsection{6/ijgc-2021-ESG0.512}

Introduction/Background* This study aimed to evaluate the diagnostic added-value of serum CA-125 \& HE4 to the International Ovarian Tumor Analysis (IOTA) Simple Rules for differentiating malignant and benign ovarian tumors preoperatively.

Methodology A Cross sectional study was conducted in the Department of Obstetrics and Gynecology on 100 patients with ovarian masses. Demographic and clinical data were prospectively collected. Histopathologic diagnosis was used as the gold standard test. Evaluation of the utility of HE4, CA125 and IOTs simple rules for preoperative identification of malignacy was based on the increment of the area under the receiver operating characteristic curve (AuROC).

Result(s)* Out of the 50 suspected benign masses as per IOTA simple rules, post surgery (considering histopathology as gold standard) $32(64 \%)$ were diagnosed as benign ovarian masses, $4(8 \%)$ borderline and $14(28 \%)$ other malignant ovarian tumors on final histopathology. Out of 50 suspected malignant masses as per IOTA simple rules, 26 (52\%) were finally diagnosed as benign, 4(8\%) borderline and $20(40 \%)$ were malignant. Sensitivity of combined testing of both tumour markers (HE4 and Ca-125) and IOTA together was calculated to be a $85.3 \%(69-95)$ whereas its specificity increased to be $94.8 \%$ (86-99). Positive predictive value was determined to $90.6 \%$ (75-98) and negative predictive value was calculated $91.7 \%$ (82-97) making its diagnostic accuracy as 91.3\% (84-96).

Conclusion* Combining IOTA Simple rules with knpown tumour markers (CA125 and HE4) increases diagnostic accuracy in predicting malignat ovarian masses preoperatively

\section{BRCA GERMLINE MUTATION FREQUENCY AND THE EFFECTS ON ONCOLOGIC OUTCOMES AMONG NORWEGIAN OVARIAN CANCER PATIENTS}

\begin{abstract}
${ }^{1}$ A Birgisdottir*, ${ }^{2} \mathrm{M}$ Bjørnslett, ${ }^{3} \mathrm{~S}$ Aaserud, ${ }^{1} \mathrm{~T}$ Paulsen, ${ }^{1} \mathrm{~A}$ Dørum. ${ }^{1}$ The Norwegian Radium Hospital, Department of Gynecological Oncology, Oslo, Norway; ${ }^{2}$ Oslo University Hospital, Department of Molecular Oncology, Oslo, Norway; ${ }^{3}$ The Norwegian Cancer Registry, Oslo
\end{abstract}

\subsection{6/ijgc-2021-ESG0.513}

Introduction/Background* Germline mutations in the BRCA1 and $B R C A 2$ genes (BRCAg) are a known risk factor for development of breast and epithelial ovarian cancer (OC). We describe the frequency of $B R C A g$ carriers, the clinical features and survival in Norwegian OC patients.

Methodology This is a prospective cohort study including OC patients at the Norwegian Radium Hospital (covering 50\% of the Norwegian population) from Jan 1st 2014 to Dec 31st 2019. Included are the patients which accepted BRCAg test after giving informed consent. DNA was isolated from peripheral blood, and mutation analyses performed with Sanger sequencing and multiplex ligation probe amplification. Data on family history, BRCAg test results was registered and data on clinical features and histopathology was collected from the department's quality database. All statistical analyses were performed using the Stata (Stata/MP 17.1) program, Chi-square 